\title{
CORRECTION
}

\section{Correction to: Challenging issues in rheumatology: thoughts and perspectives}

\author{
Nathan Lim ${ }^{1} \cdot$ Leanna Wise $^{1} \cdot$ Richard S. Panush ${ }^{1}$
}

Published online: 15 June 2021

(c) International League of Associations for Rheumatology (ILAR) 2021

\section{Correction to: Clinical Rheumatology (2021) 40:1669-1672 https://doi.org/10.1007/s10067-021-05709-4}

The original published version of this article contained an error.

In the Table 1, the erroneous decade "20210s" should be "2010s". The corrected Table 1 is shown as follow:

Table 1 Thematic issues (challenges) in modern rheumatology by decade

\begin{tabular}{ll}
\hline Decade & Theme \\
\hline $1950 \mathrm{~s}$ & Introduction of glucocorticoids \\
$1960 \mathrm{~s}$ & Establishment, development, and growth of rheumatology training programs \\
$1970 \mathrm{~s}$ & Immunology and modern science come to rheumatology \\
$1980 \mathrm{~s}$ & The silicone breast implant saga- "science on trial" \\
$1990 \mathrm{~s}$ & Documenting the value of subspecialty care and subspecialists (outcomes) \\
$2000 \mathrm{~s}$ & Quality of care and safe care \\
$2010 \mathrm{~s}$ & Cost of care; medical "waste" \\
$2020 \mathrm{~s}$ & Social justice \\
\hline
\end{tabular}

The original article can be found online at https://doi.org/10.1007/ s10067-021-05709-4.

Nathan Lim

nathan.lim@med.usc.edu

$\triangle$ Leanna Wise

leanna.wise@med.usc.edu

$\bowtie$ Richard S. Panush

panush@usc.edu

1 Division of Rheumatology, Department of Medicine, Keck School of Medicine, University of Southern California, Los Angeles County + University of Southern California (LAC+USC) Medical Center, Los Angeles, CA, USA 